\title{
The role of vector meson dominance and running masses in the hadronic contributions to the muon
}

$g-2$

\section{Tobias Goecke*}

Institut für Theoretische Physik

Universität Giessen, Heinrich-Buff-Ring 16, 35392 Giessen, Germany

E-mail: Tobias.Goecke@theo.physik.uni-giessen.de

\section{Christian S. Fischer ${ }^{\dagger}$}

Institut für Theoretische Physik

Universität Giessen, Heinrich-Buff-Ring 16, 35392 Giessen, Germany

\section{Richard Williams}

Institut für Physik

Karl-Franzens-Universität Graz, Universitätsplatz, 5, 8010 Graz, Austria

\begin{abstract}
We summarize our recent results for the quark loop part of the light-by-light scattering contribution as well as the hadronic vacuum polarisation contributions to the anomalous magnetic moment of the muon. In particular we focus on the role played by the momentum dependence of the quark- and quark-photon vertex dressing functions. We give a detailed comparison of the DysonSchwinger description of this contribution to the corresponding picture emerging from hadronic models in particular the extended Nambu-Jona-Lasonio model (ENJL). We find that the details of the momentum dependence are important on a quantitative level. Especially the transverse parts of the quark-photon-vertex, which serve as a dynamical extension of simple vector meson dominance models, do not yield the large suppression of the light-by-light contribution found in the ENJL model if realistic dressings are taken into account.
\end{abstract}

Xth Quark Confinement and the Hadron Spectrum,

October 8-12, 2012

TUM Campus Garching, Munich, Germany

* Speaker.

† Speaker. 


\section{Introduction}

In these proceedings we summarize our results on the anomalous magnetic moment of the muon presented in [1] and [2]. This quantity provides a precision test of the Standard Model (SM), the electromagnetic (EM) [3], the weak [4] and the strong force. An overview of the different contributions is given in Table 1. Clearly the QED contributions are dominant, followed by the ones from QCD. It also evident that the strong contributions dominate the uncertainty of the SM prediction. Most relevant in that respect is the leading order hadronic vacuum polarisation (LOHVP) [5]. There is some hope that this uncertainty may decrease due to improved experimental input [6] as well as the efforts of the lattice community [7, 8, 9]. In the long run the most problematic contribution may therefore be hadronic light-by-light scattering (LBL), which cannot thus far be determined in a model-independent way. It has been considered in many approaches such as the Extended Nambu-Jona-Lasinio (ENJL) model [10], the Hidden Local Symmetry (HLS) model [11], vector meson dominance (VMD) approaches [12,13], the non-local chiral quark model $(\mathrm{NL} \chi \mathrm{QM})[14,15]$, the chiral constituent quark model ( $\chi \mathrm{CQM})$ [16], in holographic models [17] and Dyson-Schwinger Equations (DSEs) $[18,19,1]$. The lattice calculations of LBL are still at an exploratory stage [20]. The combined theory result quoted in Table 1 is a number that different groups agreed upon [21] by combining several hadronic models. For comparison, we list here the most recent experimental result from Refs. [22, 23] which reveals a discrepancy of about three standard deviations [5]; very recent results even indicate a significance ranging between 4.7 and $4.9 \sigma[24]$.

These results are exciting since they may provide a signal of new physics beyond the Standard Model. However, as we will argue in detail in the course of this work they should be taken with some caution. The present estimate of the hadronic light-by-light contribution including its potential error has been derived from model calculations. Although much care has been invested in the error estimate, we believe that the inherent limitations of the models employed may have led to an overly optimistic value. Here we summarise our comparison between our approach and that of models for one of the more contentious contributions to LBL [1], in order to highlight this point. To this end we discuss the hadronic photon four-point function and summarize the DSE approach

\begin{tabular}{c||r|c|l} 
Contribution & $a_{\mu} \times 10^{11}$ & $\frac{a_{\mu}^{i}}{a_{\mu}^{S M}}$ & $\left(\frac{\delta a_{\mu}^{i}}{\delta a_{\mu}^{S M}}\right)^{2}$ \\
\hline \hline QED & $116584718.1(0.2)$ & $99.99390 \%$ & $00.00098 \%$ \\
\hline weak & $153.2(1.8)$ & $00.00013 \%$ & $00.07910 \%$ \\
\hline QCD LOHVP & $6949.1(58.2)$ & $00.00596 \%$ & $82.69628 \%$ \\
\hline QCD HOHVP & $-98.4(1.0)$ & $00.00008 \%$ & $00.02441 \%$ \\
\hline QCD LBL & $105(26)$ & $00.00009 \%$ & $16.50391 \%$ \\
\hline Standard Model & $116591827.0(64)$ & $100 \%$ & $100 \%$ \\
\hline Experiment & $116592089(63)$ & & \\
\hline \hline Exp-Theo & $262(89)$ & &
\end{tabular}

Table 1: Standard Model contributions to the muon $g-2$. 


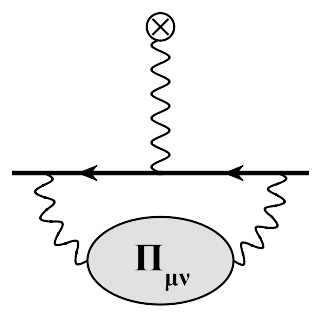

(a)

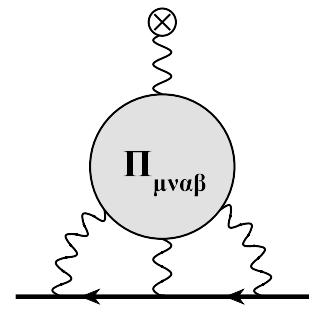

(b)

Figure 1: The two classifications of corrections to the photon-muon vertex function: (a) hadronic vacuum polarization contribution to $a_{\mu}$. The vertex is dressed by the vacuum polarization tensor $\Pi_{\mu v}$; (b) the hadronic light-by-light scattering contribution to $a_{\mu}$.

used in this work in Sections 2 and 3. Then we shortly discuss the results for the leading order hadronic contribution in Section 4. In Section 5 we compare our method to the extended NambuJona-Lasinio model. We explain similarities and differences and how these affect the results for the quark-loop light-by-light contribution in Section 6. We conclude in Section 7.

\section{Basics}

On mass-shell, the muon-photon vertex can be written in terms of two momentum dependent form-factors

$$
\nearrow^{\otimes}=\bar{u}\left(p^{\prime}\right)\left[F_{1}\left(q^{2}\right) \gamma_{\alpha}+i F_{2}\left(q^{2}\right) \frac{\sigma_{\alpha \beta} q^{\beta}}{2 m_{\mu}}\right] u(p),
$$

where $p$ and $p^{\prime}$ are the muon momenta, $q$ is the photon momentum and $\sigma_{\alpha \beta}=\frac{i}{2}\left[\gamma_{\alpha}, \gamma_{\beta}\right]$. The anomalous magnetic moment is defined in the limit of vanishing photon momentum, $q^{2}$, as

$$
a_{\mu}=\frac{g-2}{2}=F_{2}(0) .
$$

The HVP contribution, shown in Fig. 1(a) is defined within the DSE approach as the hadronic photon self energy

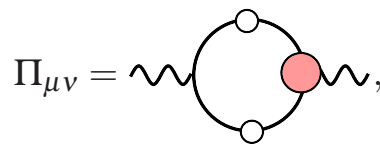

which is itself defined in terms of the full quark and the quark-photon vertex. This representation is exact, up to truncations of vertex and propagator. This quantity is well described within our approach as is detailed in section 4 .

The LBL contribution to this vertex is depicted in Fig. 1(b). This hadronic photon four-point function can be split into several parts, which we organize by the number of quark T-Matrices 


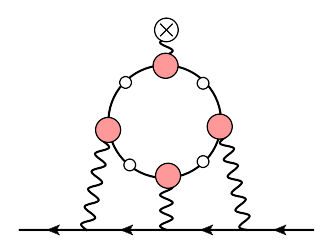

Figure 2: The quark loop contribution to the muon $g-2$. The quarks and vertices are fully dressed.

involved

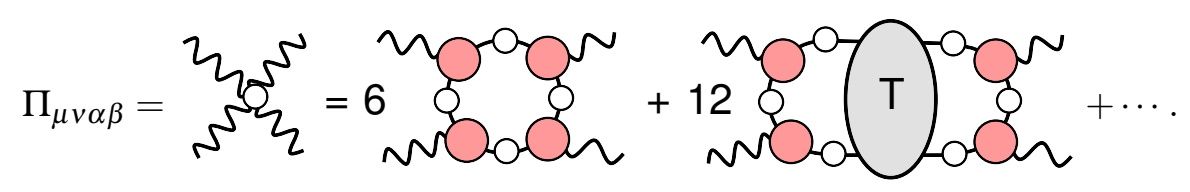

The pre factors indicate the number of permutations that must be computed. The second term, containing just one T-matrix, has been considered in Refs. [18, 19] by performing a pseudoscalar resonant expansion. We found good agreement with corresponding results from hadronic models $[10,25,26,27,28,12,13,29,21,14,30,16]$. The ellipsis contains, for example, contributions that can be written as charged pion loops including also the pion polarizabilities. While in earlier works these contributions have been argued to be small, a recent work suggests substantial contributions to LBL which need to be evaluated carefully in the future [31]. Note, however, that within the approximation of QCD, we are working with (see Sec. 3) the representation in Eq. (2.4) is consistent with the exact representation Eq. (2.3), a fact that is interesting when it comes to considerations about systematic uncertainties.

Later we concentrate on the first part of Eq. (2.4) referred to here as the quark-loop. It consists of four fully dressed quark propagators as well as four fully dressed quark-photon vertices. Its contribution to $a_{\mu}$ is shown explicitly in Fig. 2. The focus of these proceedings will be in the transverse parts of these fully-dressed vertices, which in principle contain contributions from vector mesons. It has been shown that these contributions generate a strong suppression of the quark-loop contribution to $a_{\mu}$ in the ENJL model [10]. Below we will argue that this suppression is in fact a model artefact attributable to the contact interaction featured therein.

\section{Method}

First, let us briefly review the needed Dyson-Schwinger equations and their truncation. The first ingredient for the calculation of the quark-loop is the quark propagator, $S(p)$, whose inverse is described by the DSE

$$
S^{-1}(p)=Z_{2} S_{0}^{-1}+g^{2} Z_{1 f} \frac{4}{3} \int \overline{d k} \gamma^{\mu} S(k) \Gamma^{v}(k, p) D_{\mu v}(q),
$$

To solve this equation we require the dressed gluon propagator $D_{\mu v}(p)$ and the dressed quarkgluon vertex $\Gamma_{v}(k, p)$. In the rainbow truncation employed here we use a combined ansatz for both of these quantities retaining only the $\gamma_{v}$ component of the vertex. This truncation has been proposed in Refs. [32, 33] and has achieved phenomenological success in describing meson properties such 
as masses, decay constants and electromagnetic form factors $[32,33,34,35,36]$ as well as various baryon properties $[37,38,39,40]$.

The second ingredient for the calculation of the quark-loop is quark-photon vertex, $\Gamma_{\mu}$, determined from the inhomogeneous equation

$$
\left[\Gamma_{\mu}(P, k)\right]_{r s}=Z_{1} \gamma_{\mu}-Z_{2}^{2} \frac{4}{3} \int \overline{d q}\left[S\left(q_{+}\right) \Gamma_{\mu}(P, q) S\left(q_{-}\right)\right]_{u t} K_{t u, r s}(k-q),
$$

where $K_{t u, r s}(k-q)$ is the quark-antiquark interaction-kernel. To satisfy the vector- and axial-vector Ward-Takahashi identities we choose the ladder-exchange kernel, consistent with rainbow truncation of the quark DSE above. An important consequence of this truncation is the dynamical generation of vector meson poles in the transverse components. Thus the tenets of vector meson dominance models (VMD) are automatically encoded on the level of interacting quarks and gluons. Note furthermore that the vertex from Eq. (3.2) and the quark from Eq. (3.1) fulfill the Ward-Takahashi identity (WTI)

$$
\mathrm{i} P_{\mu} \Gamma_{\mu}(P, k)=S^{-1}\left(k_{-}\right)-S^{-1}\left(k_{+}\right) .
$$

Both properties are crucial for the realistic description of electromagnetic properties, e.g. the pion charge radius [34]. Their relevance to the leading order hadronic vacuum polarisation contribution to $a_{\mu}$ has been highlighted in Ref. [2], see Sec. 4. We will see below also the importance of the correct description of the transverse structures as regards the hadronic LBL contribution. From this we will argue that the twenty percent error quoted in Table 1 is too optimistic since it is obtained from approximations that implicitly miss information that we later find to be important.

\section{The leading order QCD contribution}

In the following we summarize our results for the leading order hadronic contribution, the leading order hadronic vacuum polarisation published in [2]. This quantity is a very important test for any approach that aims at a quantitative prediction of the much more complicated LBL. In short, using the quark [Eq. (3.1)] and the quark-photon vertex [Eq. (3.2)] we calculate this contribution via Eq. (2.3). Our result is

$$
a_{\mu}^{L O H V P, D S E}=(6760-7440) \times 10^{-11},
$$

where the given range reflects our systematic model uncertainty, see [2] for details. Our result is close to the rather model independent analysis from Ref. [5] , i.e. $6949 \times 10^{-11}$. This is a deviation of less then ten percent. Although this precision is not a challenge for the model-independent HVP predictions, a corresponding precision for LBL would be a great breakthrough. Furthermore, in Ref. [2] we presented a determination of the Adler function, that can be obtained from the hadronic vacuum polarisation tensor, c.f. Eq. (2.3), that shows good agreement with model-independent results from dispersion relations on all scales. What is especially interesting in the context of these proceedings is that we found the transverse part of the quark-photon vertex to yield the dominant contribution of $\sim 80 \%$ to $a_{\mu}^{L O H V P}$. Since this part contains a dynamically generated vector meson bound state, this explains why VMD approximations work reasonably well for HVP, see e.g. [41]. 


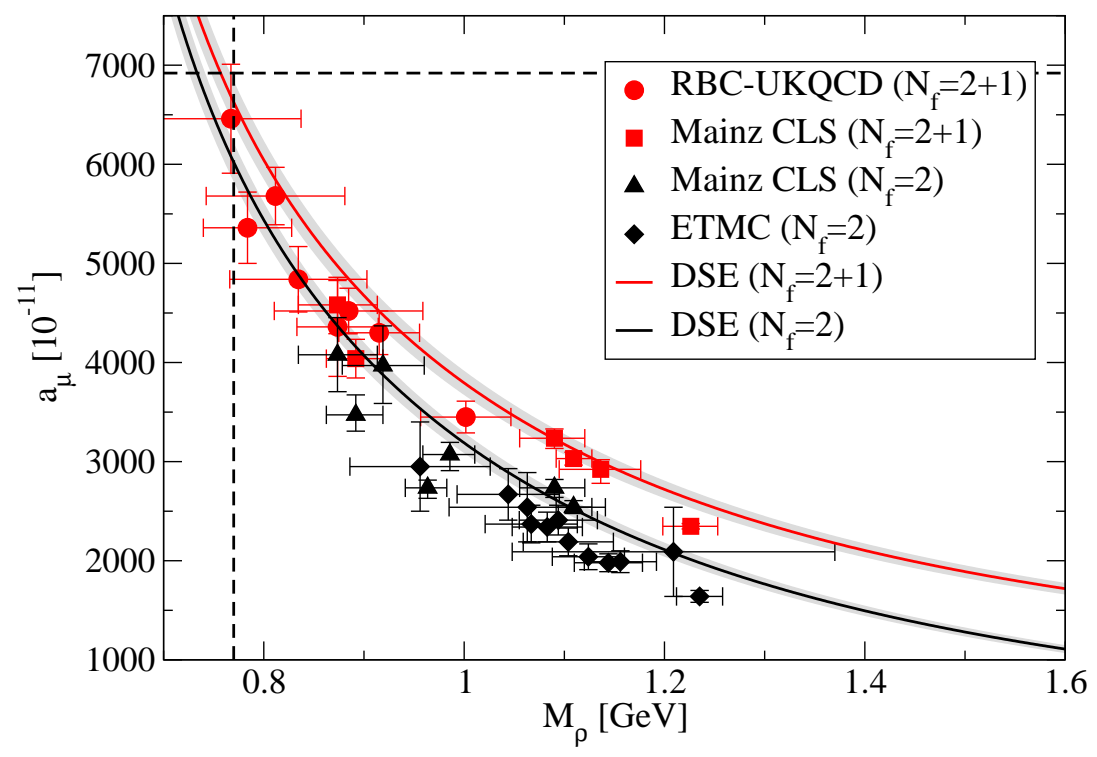

Figure 3: The LOVHP contribution to $a_{\mu}$ as a function of the $\rho$ mass. We compare our results for the $N_{f}=2$ (black) and $N_{f}=2+1$ (red) flavour cases to lattice results. The grey bands represent our numerical uncertainty.

Models that miss these physics have to use a constituent quark mass much lower than expected $[16,42]$.

In Ref. [2] we also compared our results against lattice QCD. Here, we present an updated comparison including more recent lattice data in Fig. 3. Both, the lattice and the DSE calculations are performed at various current quark masses. In order to provide a gauge and scheme invariant comparison between the approaches we have plotted the results against the corresponding varying mass of the $\rho$-meson. In the figure, we compare against results from Refs. [7, 8, 43] for the cases of $N_{f}=2$ (black) and $N_{f}=2+1$ (red) flavours. We find agreement within error bars, showing nicely that the DSEs have the correct physics included and that the $\rho$ meson mass is the important scale here.

It is interesting, that while we confirm the simple picture of VMD for the case of the leading hadronic contribution, the picture will be very different in the case of the LBL contribution. This is the subject in the following.

\section{Comparison: DSE vs. ENJL}

To explicate the differences between our approach and others, we make a comparison between the DSE truncation used in this work and the ENJL model. The first striking difference lies in 
the fact that DSEs have a smooth ultraviolet limit, wherein at large momenta we connect with perturbation theory. ENJL on the other hand is non-renormalisable and features an effective cutoff scale on the order of a GeV. This necessitates the splitting of the photon four-point function into a high- and low-energy region that is not unique due to their being two independent scales. Additionally, the dressings of the ENJL model feature trivial momentum dependencies due to its contact interaction. In this regard the DSE approach is quite different. In what follows we will explore this is some detail; further information can be found in Ref. [1].

Primarily we will focus on the two flavour case as this is sufficient to make our point. However, we will quote $N_{f}=4$ results for completeness in the later sections.

\subsection{The ENJL perspective}

The discussion here is based on Refs. [10, 44], where further details may be found. In the ENJL model, the quark propagator assumes the form of a momentum independent constituent quark

$$
S_{\mathrm{ENJL}}^{-1}(p)=-i \not p+M,
$$

with a typical mass of $M \approx 300 \mathrm{MeV}$. The wave function renormalisation, $Z_{f}$, is unity. The quarkphoton vertex is given by the bubble sum

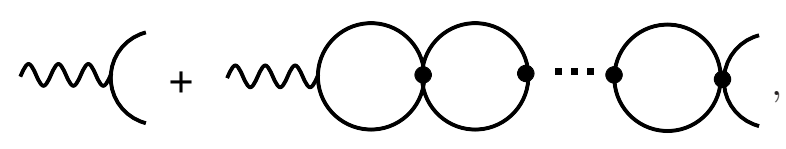

which can be resummed as a geometric series $\sum_{n}$ Bubble $^{n}=1 /(1-$ Bubble $)$. This simple behaviour is due to the effective contact interaction that decouples the loop integrals. Consequently, the result depends only on the total photon momentum $Q$ and has no dependence on the relative quark momentum. Explicitly the vertex has the form

$$
\Gamma_{\mu}^{\mathrm{ENJL}}=\gamma_{\mu}-\gamma_{\mu}^{T} \frac{Q^{2}}{Q^{2}+M_{V}^{2}},
$$

which contains the bare vertex $\gamma_{\mu}$ and the leading transverse structure $\gamma_{\mu}^{\mathrm{T}}=\left(\delta_{\mu v}-Q_{\mu} Q_{v} / Q^{2}\right) \gamma_{v}$. The dressing of this transverse part is given here in the VMD limit of the ENJL model [44] where for two flavours $M_{V}$ is identified with the $\rho$-mass. Using the transversality of the hadronic photon four-point function with respect to its photon legs, this vertex can be reduced to $\gamma_{\mu} M_{V}^{2} /\left(Q^{2}+M_{V}^{2}\right)$ which highlights the strong suppression induced by the VMD contribution to the vertex. Note that this model is consistent with the Ward-Takahashi identity, with the $\gamma_{\mu}$ component the constrained gauge-part and $\gamma_{\mu}^{T}$ the transverse part.

\subsection{The DSE perspective}

Now we investigate the corresponding objects in the case of DSEs. The full inverse quark propagator is given by

$$
S_{\mathrm{DSE}}^{-1}(p)=Z_{f}^{-1}\left(p^{2}\right)\left(-i \not p+M\left(p^{2}\right)\right)
$$


which contains two momentum dependent dressing functions $Z_{f}\left(p^{2}\right)$ and $M\left(p^{2}\right)$, which correspond to the wavefunction renormalisation and the mass function, respectively. The quark-photon vertex is given as a sum of gluon ladders

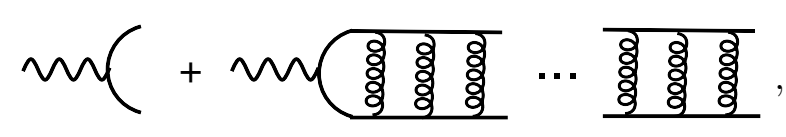

which, as opposed to Eq. (5.3), can not be resummed as a trivial series and must instead be solved numerically. In general, this vertex can be decomposed into twelve tensor components

$$
\Gamma_{\mu}(Q, k)=\sum_{i=1}^{4} \lambda^{(i)}(Q, k) L_{\mu}^{(i)}+\sum_{i=1}^{8} \tau^{(i)}(Q, k) T_{\mu}^{(i)},
$$

where $k$ is the relative quark momentum and $Q$ the total photon momentum. Note that the first part, containing the $\lambda^{(i)}$, is also called the Ball-Chiu vertex $\Gamma_{\mu}^{B C}$ [45]. The vector meson bound state does also appear in the transverse vertex structure in the DSE/BSE case. This is most easily pictured in the form of a simple fit [34] to the numerical results of the quark-photon vertex

$$
\Gamma_{\mu}(Q, k) \simeq \Gamma_{\mu}^{\mathrm{BC}}-\gamma_{\mu}^{\mathrm{T}} \frac{\omega^{4} N_{V}}{\omega^{4}+k^{4}} \frac{f_{V}}{M_{V}} \frac{Q^{2}}{Q^{2}+M_{V}^{2}} e^{-\alpha\left(Q^{2}+M_{V}^{2}\right)},
$$

which consists of the non-transverse Ball-Chiu part, $\Gamma_{\mu}^{\mathrm{BC}}$, and the leading transverse structure corresponding to $T_{\mu}^{1}=\gamma_{\mu}^{\mathrm{T}}$. For the parameters we find reasonable agreement with the numerical solution with $\omega=0.66 \mathrm{GeV}, \alpha=0.15$ and $N_{V} f_{V} / M_{V}=0.152$. Note that, as in the ENJL model, we have in Eq. (5.7) a part that is given via the WTI (the BC vertex) and a transverse part. We wish to emphasize once more, that the vector meson pole appearing in the transverse part is generated dynamically in the DSE and ENJL approaches.

\subsection{Differences between DSEs and ENJL model}

We already stated that one of the chief differences between the DSE and ENJL approach lies with the momentum dependencies of the propagators and vertices. We compare the quark propagators in Fig. 4, where one sees a non-trivial momentum dependence in both the wave function renormalisation and the mass function for the DSE. In particular, the mass function features the
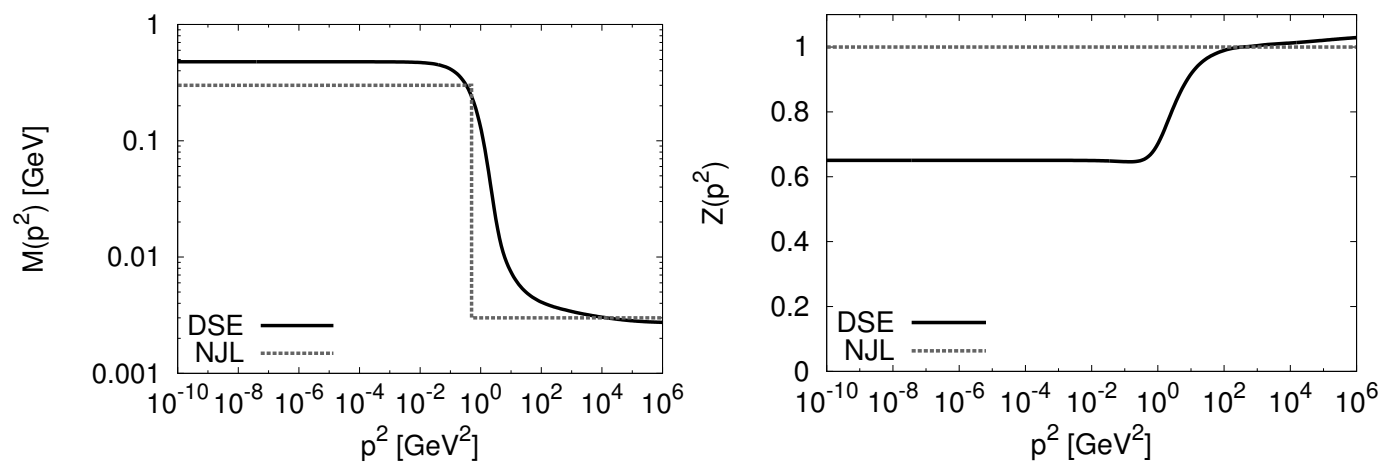

Figure 4: Comparison of the mass function $M$ and the wavefunction $Z_{f}$ for DSE and ENJL quarks. 
expected resumed one-loop perturbative behaviour at large momenta and smoothly connects to the infrared domain where a constituent-like mass is dynamically generated. This rapid accumulation of mass is consistent with results of the operator product expansion [46]. Indeed, the same qualitative behaviour is seen on the lattice, see Ref. [47] for a comparison. In contrast, the dressing functions in the ENJL model are just constants and hence miss essential features present in QCD.

Next we compare the approximate functional forms of the quark-photon vertex in the ENJL model, Eq. (5.3) and DSE approach, Eq. (5.6). The dressing of the $\gamma_{\mu}$ part is shown in Fig. 5. Consistent with the Ward-Takahashi identity it is unity in the ENJL case due to the quark wavefunction being trivial there. In contrast, the corresponding vertex dressing in the DSE approach behaves as $\lambda^{(1)} \sim 1 / Z_{f}$, owing again to Ward identities. A consequence of the wavefunction being screened
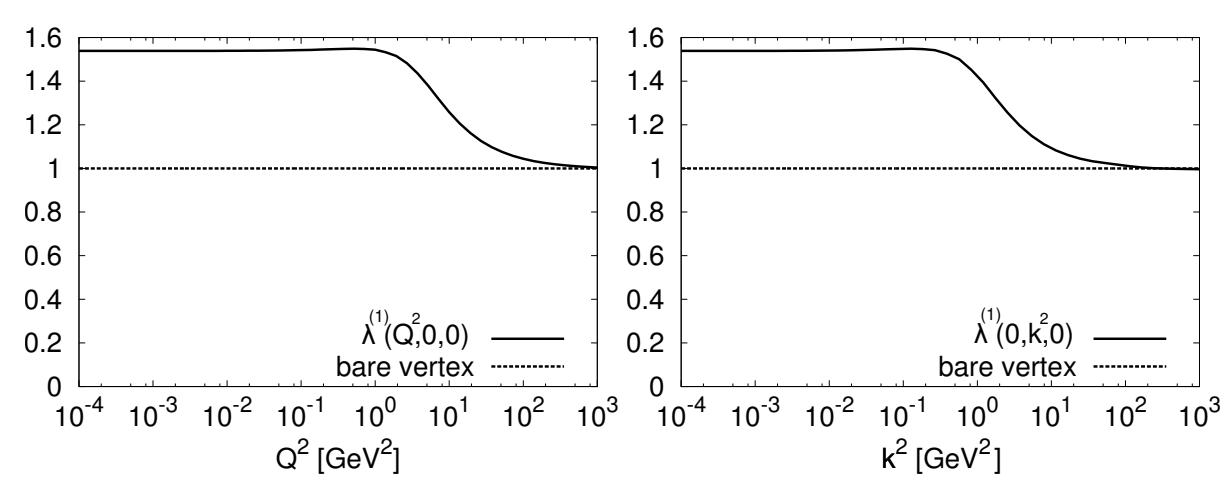

Figure 5: The leading $\lambda^{(1)}$ component of the quark-photon vertex constrained by the Ward-Takahashi identity of Eq. (3.3). We show two slices with relative, $k$, and total, $Q$, momentum set to zero, respectively. The constant dressing corresponds to the ENJL model.

for a large part of the momentum regime is that $\lambda^{(1)}>1$ leading to an enhancement. However, since each vertex can be paired with a quark propagator that features a screening $\sim Z_{f}$ these effects essentially cancel in the quark-loop contribution to $a_{\mu}$. Thus, quantitative differences between the ENJL model and the DSE approach owing to non-trivial wavefunction renormalisation and the $\lambda^{(1)} \gamma_{\mu}$ part of the vertex are expected to be small. This is confirmed in explicit calculations below.

Now we consider the transverse part of the vertex, $\tau^{(1)}=\gamma_{\mu}^{\mathrm{T}}$. We compare the fit function of Eq. (5.7) with the full numerical solution, $\tau_{\text {calc }}^{(1)}$, of the vertex BSE in Fig. 6 for a momentum slice in which the relative momentum between the two quarks is vanishing. We see that at small photon momentum, $Q$, qualitative agreement between the ENJL and DSE calculations. Since the ENJL model is not ultraviolet complete, it is not surprising that large differences arise at large values of the photon momentum. Fortunately, under the integral such a momentum region is not weighted strongly in the determination of $a_{\mu}$ and so this deviation is not important. This situation is drastically changed when one takes into account the relative momentum between the two quarks, i.e. $k^{2} \neq 0$. We see from the fit to the DSE calculation of the vertex, Eq. (5.7) that the transverse part of the vertex is highly suppressed for $k^{2}>\omega^{2}$. The ENJL model presents no such dependence. The quantitative impact of this damping will be discussed in the next section. 


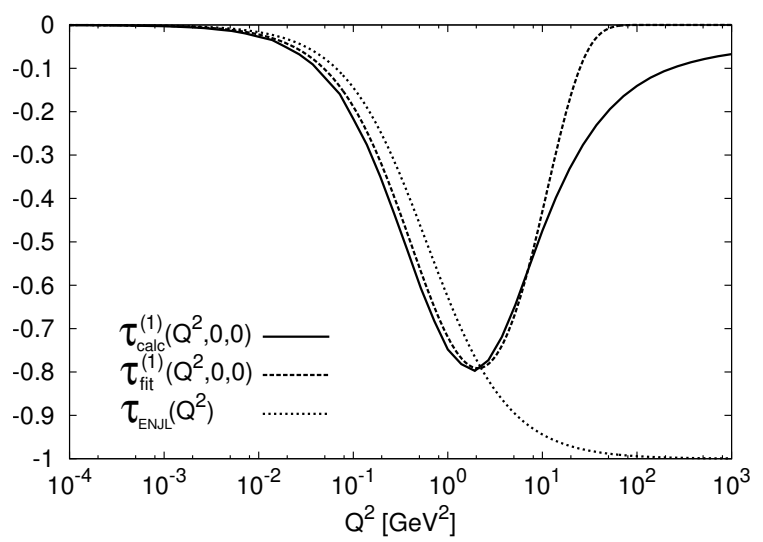

Figure 6: The dependence of the dominant transverse dressing $\tau^{(1)}$ on the space-like photon momentum $Q^{2}$ is shown for the explicit solution of the quark-photon vertex BSE, Eq. (3.2), the fit to this given in Eq. (5.7), and the transverse part of the ENJL vertex, Eq. (5.3).

\section{The hadronic light-by-light contribution}

We are now ready to turn the qualitative discussion in the last section into quantitative statements. To this end we give a summary of the results of Ref. [1] on the contribution to $a_{\mu}^{\mathrm{LBL} \text {, quark-loop }}$ using various quark- and vertex-dressings. For some quantities we calculate the averages of dressing functions as the arithmetic mean from samples of the VEGAS Monte-Carlo routine [48] we

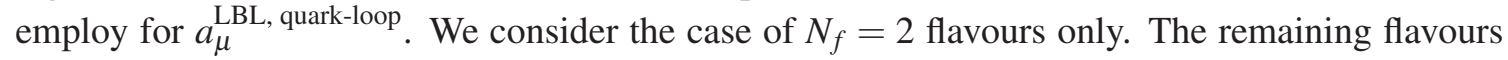
constitute a correction of the order of ten percent and don't change the general picture that emerges from this comparison.

\subsection{Influence of the quark and gauge part of the vertex}

Here, we explicitly show that the suspected cancellation between the wavefunction renormalisation, $Z_{f}$, of the quark propagator and the leading gauge part of the quark-photon vertex does, indeed, take place in the calculation of the quark-loop contribution to $a_{\mu}$. Since the dressing of the quark photon vertex is directly related via the Ward-Takahashi identity to $Z_{f}$ it suffices to perform the calculation in full, and with $Z_{f}=1 / Z_{2}{ }^{1}$. We display the results in Table 2 . As is evident

\begin{tabular}{l|c} 
& $a_{\mu}^{L B L, q l}\left[10^{-11}\right]$ \\
\hline$M, Z_{f}, \lambda^{(1)}$ all dynamical & 100 \\
$M$ dynamical, $Z_{f}=1 / Z_{2}, \lambda^{(1)}=Z_{2}$ & 102 \\
$M=0.2 \mathrm{GeV}, Z_{f}=1 / Z_{2}, \lambda^{(1)}=Z_{2}$ & 104
\end{tabular}

Table 2: The quark-loop contribution $a_{\mu}^{L B L, q l}$ to hadronic LBL. Compared are our result for full quark propagator and gauge part of the vertex with an approximation where the momentum dependence of the quark wave function is neglected. In addition we show the result where also the quark mass is replaced by a constant $M=0.2 \mathrm{GeV}$.

\footnotetext{
${ }^{1}$ The $Z_{2}$ is a necessary renormalisation constant to ensure multiplicative renormalizability
} 
by the similarity of the two results, this clearly supports our qualitative discussion regarding the cancellation.

In the process of performing these integrals, we also determined the average mass probed to be $\sqrt{\left\langle M^{2}\right\rangle} \approx 0.2 \mathrm{GeV}$, as weighted by the Monte-Carlo integration. While this finding explains why constituent quark models such as Ref. [16] and [49] must necessarily use very light constituent quark masses in order to achieve reasonable results, it also shows that this definition of effective mass is process dependent, contrary to the running mass function defined through the quark DSE, which is universal. The projection onto a certain process is only introduced through the process dependent Feynman-diagrams which includes the quark and thus the mass function.

We wish to emphasize that the $\lambda^{(1)} \gamma_{\mu}$ part is only one of three terms in the gauge part of the vertex. Although the corresponding result is already approximately gauge invariant [1], the functions $\lambda^{(2 / 3)}$ from Eq. (5.6) have to be included for principal reasons. Unfortunately the corresponding numerics turns out to be delicate [19], such that more work is needed to resolve this issue.

\subsection{Influence of the vertex: transverse part}

Now we investigate the impact of the leading transverse part of the vertex, $\gamma_{\mu}^{\mathrm{T}}$, on the quarkloop contribution to $a_{\mu}$.

Since the ENJL model does not feature a relative momentum dependence in its vertex dressings, we compare the ENJL result for the transverse vertex with our DSE determinations where we force the condition $k^{2}=0$. From the left-panel in Table 3 we see agreement between the results which highlights that the differences at large momenta in the fit functions are largely irrelevant.

In the right-panel of the same table, we show the corresponding results for when the relative quark momentum is taken into account. An appropriate function is also introduced to the ENJL parametrisation

$$
f\left(k^{2}\right)=\frac{\omega^{4}}{k^{4}+\omega^{4}},
$$

with $\omega$ defined above. Again, we see a degree of parity between the models but note that there is more than a factor of two difference as compared to the results with restricted momentum dependence.

\begin{tabular}{l|c} 
Vertex Dressing, $k^{2}=0$ & $a_{\mu}^{L B L, q l}\left[10^{-11}\right]$ \\
\hline \hline$\gamma_{\mu} \lambda^{(1)}+\gamma_{\mu}^{\mathrm{T}} \tau_{\text {ENJL }}$ & 43 \\
\hline$\gamma_{\mu} \lambda^{(1)}+\gamma_{\mu}^{\mathrm{T}} \tau_{\text {fit }}^{(1)}$ & 43 \\
\hline$\gamma_{\mu} \lambda^{(1)}+\gamma_{\mu}^{\mathrm{T}} \tau_{\text {calc }}^{(1)}$ & 41 \\
\hline
\end{tabular}

\begin{tabular}{l|c} 
Vertex Dressing, $k^{2} \neq 0$ & $a_{\mu}^{L B L, q l}\left[10^{-11}\right]$ \\
\hline \hline$\gamma_{\mu} \lambda^{(1)}+\gamma_{\mu}^{\mathrm{T}} \tau_{\text {ENJL } f\left(k^{2}\right)}$ & 103 \\
\hline$\gamma_{\mu} \lambda^{(1)}+\gamma_{\mu}^{\mathrm{T}} \tau_{\text {fit }}^{(1)}$ & 105 \\
\hline$\gamma_{\mu} \lambda^{(1)}+\gamma_{\mu}^{\mathrm{T}} \tau_{\text {calc }}^{(1)}$ & 96
\end{tabular}

Table 3: Leading gauge part and leading transverse vertex component, with dressing functions from the ENJL model, VMD like fit from DSE/BSE $\left(\tau_{\mathrm{fit}}^{(1)}\right)$, and from an explicit calculation of the quark-photon DSE $\left(\tau_{\text {calc }}^{(1)}\right)$. Results are shown without (left table), and with (right table) the inclusion of a dependence on the relative momentum.

Our most sophisticated vertex construction is the leading gauge part of the vertex plus the leading transverse part, corresponding to the last line of the right Table 3. Repeating this calculation 
for the case of $N_{f}=4$ flavours we obtain

$$
a_{\mu}^{L B L, q l, N_{f}=4}=(107 \pm 2) \times 10^{-11}
$$

where the error is purely statistical.

\section{Summary and conclusions}

We summarised the detailed comparison of the DSE framework to the ENJL model with respect to the quark loop part of the hadronic light-by-light contribution to the anomalous magnetic moment of the muon, $a_{\mu}^{L B L, q l}$, presented in Ref. [1]. We found similarities but also important differences. Our main focus was on the influence of the momentum dependence of dressing functions that are not present in the simplified treatment within the ENJL model. These are the quark mass and wave functions as well the leading non-transverse and transverse dressing functions of the quark-photon vertex. Since some of the momentum dependencies are related via a Ward-Takahashi identity they cancel approximately in the calculation of $a_{\mu}^{L B L, q l}$. Other important differences, however, remain. One is the impact of the running quark mass function, which turns out to be equivalent to an effective constituent quark mass of $M \approx 0.2 \mathrm{GeV}$, considerably smaller than typical values considered within the ENJL model. This should, however, not be understood as a general definition of a constituent mass, but rather a way how to make a complicated integration simpler to get some intuitive insight into the physics and numerics at work. The other important difference concerns the impact of the transverse parts of the vertex. In the ENJL model, the dependence of this part of the vertex on the relative momentum $k$ between the quarks is neglected resulting in a large decrease of $a_{\mu}^{L B L, q l}$ due to transverse contributions. Within the DSE approach we have shown that this suppression is dramatically reduced when the full momentum structure of the vertex is taken into account.

In Table 4 we compare our current results for the different contributions to $a_{\mu}^{L B L}$ with the ENJL-results of Ref. [10], the current 'standard' result of Ref. [21] (summarising calculations from different sources) and the values from a recent estimate within a chiral quark model [16], which has been advocated as a qualitative reference. Compared to the ENJL-model we nicely agree in

\begin{tabular}{l||c|c|c|c||c}
{$\left[10^{-11}\right]$} & $a_{\mu}^{L B L,\left(\pi_{0}\right)}$ & $a_{\mu}^{L B L,\left(\eta, \eta^{\prime}\right)}$ & $a_{\mu}^{L B L, q l}$ & $a_{\mu}^{L B L, o t h e r}$ & $a_{\mu}^{L B L}$ \\
\hline ENJL,Ref.[10] & \multicolumn{2}{|c|}{$85(13)$} & $21(3)$ & $-23(16)$ & $83(32)$ \\
\hline standard,Ref.[21] & \multicolumn{3}{|c|}{$116(13)$} & $-11(13)$ & $105(26)$ \\
\hline$\chi$ QM,Ref.[16] & 68 & - & 82 & - & 150 \\
\hline DSE, Ref. [1] & $58(1)$ & $23(1)$ & $107(2)$ & - & $188(4)(90)$ \\
\hline
\end{tabular}

Table 4: Selected partial and total results for $a_{\mu}^{L B L}$. In the first line we display the ENJL result, the second line displays the current 'standard result' (numerical and systematic error), the third line stems from a recent estimate within a chiral quark model [16] (evaluated at a constituent mass of $M=240 \mathrm{MeV}$ ), whereas the fourth line shows our results so far (numerical error only, with a guess of the systematic error of the total result). Cells with a dash mark contributions which have not (yet) been calculated in the corresponding approach. The column 'other' sums up contributions from scalar and axialvector meson exchange as well as contributions from dressed pion loops. 
the meson exchange contributions $a_{\mu}^{L B L,\left(\pi_{0}\right)}$ and $a_{\mu}^{L B L,\left(\eta, \eta^{\prime}\right)}$ but note a drastic enhancement of the quark-loop contribution. We have explained this difference above. The same arguments apply to the results of the summary of Ref. [21] in the second line. The comparison with the chiral quark model $(\chi \mathrm{QM})$ in the third line is interesting. They have applied a constituent mass in the range of $M=230-250 \mathrm{MeV}$. As we have argued above, our results with full momentum dependence are reproduced by an approximation using an average quark mass function of $\langle M\rangle=200 \mathrm{MeV}$, i.e. even lower. For such a small value the results of Ref.[16] become smaller in the pion exchange channel but larger in the quark-loop contribution resulting in a total of $168 \times 10^{-11}$ in very good agreement with our full results.

Small constituent quark masses of order $\sim 200 \mathrm{MeV}$ have been found to be necessary in various approaches using simple models, see e.g. Refs. [50, 42, 49]. As argued above, our findings can explain this on a much deeper level of sophistication. It has to be mentioned, however, that not all models agree on whether the quark-loop- and meson-exchange-contribution are two ways to describe the same contribution, or whether these have to be added. From the viewpoint of QCD diagrams it seems clear that these are completely separate contributions, see e.g our Ref [19].

We conclude from our study that the standard value $a_{\mu}^{L B L}=105(26) \times\left[10^{-11}\right]$ used in current evaluations of the anomalous magnetic moment of the myon $[5,24]$ may be too small concerning its central value and is probably much too optimistic in its error estimate. We emphasize once again the importance of taking explicit momentum dependencies into account, a task that the DSE framework is well capable for. Our current results for $a_{\mu}^{L B L}$ are preliminary, since we neglected contributions from the transverse and non-transverse parts of the quark-photon vertex in the quarkloop. We are working on this issue. We are also working on an explicit calculation of contributions from the charged pion-loop which, according to the study of [31] may be more important than previously thought, see also the contribution of Ramsey-Musolf to these proceedings [51].

To compete with the expected error bars of the forthcoming experiments we need to improve the theoretical error of $a_{\mu}^{L B L}$ into the twenty, or even better into the ten percent region. Within the DSE framework this level has been reached in the case of hadronic vacuum polarisation [2], where nice agreement with corresponding lattice calculations has been obtained. For hadronic light-bylight we propose a similar strategy. We believe that only the systematic comparison of different methods like effective models, the DSE framework and lattice gauge theory provides the potential for a reliable calculation of $a_{\mu}^{L B L}$.

Acknowledgments

This work was supported by the DFG under contract FI 970/8-1 and the Austrian Science Fund FWF under project M1333-N16.

\section{References}

[1] T. Goecke, C. S. Fischer and R. Williams, Phys. Rev. D in press, arXiv:1210.1759 [hep-ph].

[2] T. Goecke, C. S. Fischer, and R. Williams Phys.Lett. B704 (2011) 211-217, arXiv: 1107.2588 [hep-ph].

[3] T. Aoyama, M. Hayakawa, T. Kinoshita, and M. Nio arXiv:1205.5370 [hep-ph]. 
[4] A. Czarnecki, W. J. Marciano, and A. Vainshtein Phys.Rev. D67 (2003) 073006, arXiv:hep-ph/0212229 [hep-ph].

[5] K. Hagiwara, R. Liao, A. D. Martin, D. Nomura, and T. Teubner J.Phys.G G38 (2011) 085003 , arXiv:1105.3149 [hep-ph].

[6] F. Jegerlehner and A. Nyffeler Phys.Rept. 477 (2009) 1-110, arXiv:0902.3360 [hep-ph].

[7] X. Feng, K. Jansen, M. Petschlies, and D. B. Renner Phys.Rev.Lett. 107 (2011) 081802, arXiv:1103.4818 [hep-lat].

[8] P. Boyle, L. Del Debbio, E. Kerrane, and J. Zanotti arXiv:1107.1497 [hep-lat] .

[9] M. Della Morte, B. Jager, A. Juttner, and H. Wittig JHEP 1203 (2012) 055, arXiv: 1112.2894 [hep-lat].

[10] J. Bijnens, E. Pallante, and J. Prades Nucl.Phys. B474 (1996) 379-420, arXiv : hep-ph/ 9511388 [hep-ph].

[11] M. Hayakawa, T. Kinoshita, and A. Sanda Phys.Rev.Lett. 75 (1995) 790-793, arXiv:hep-ph/9503463 [hep-ph].

[12] M. Knecht and A. Nyffeler Phys.Rev. D65 (2002) 073034, arXiv : hep-ph/0111058 [hep-ph].

[13] K. Melnikov and A. Vainshtein Phys.Rev. D70 (2004) 113006, arXiv: hep-ph/ 0312226 [hep-ph].

[14] A. E. Dorokhov and W. Broniowski Phys.Rev. D78 (2008) 073011, arXiv: 0805.0760 [hep-ph].

[15] A. Dorokhov, A. Radzhabov, and A. Zhevlakov arXiv:1204.3729 [hep-ph] .

[16] D. Greynat and E. de Rafael, JHEP 1207 (2012) 020 arXiv:1204.3029 [hep-ph] .

[17] L. Cappiello, O. Cata, and G. D’Ambrosio Phys.Rev. D83 (2011) 093006, arXiv: 1009.1161 [hep-ph].

[18] C. S. Fischer, T. Goecke, and R. Williams Eur.Phys.J. A47 (2011) 28, arXiv: 1009.5297 [hep-ph].

[19] T. Goecke, C. S. Fischer, and R. Williams Phys.Rev. D83 (2011) 094006, arXiv: 1012.3886 [hep-ph ], Erratum-ibid. D86 (2012) 099901.

[20] T. Blum, M. Hayakawa and T. Izubuchi, arXiv:1301.2607 [hep-lat] author = "Blum, T. and Hayakawa, M. and Izubuchi, T.", title = "Hadronic corrections to the muon anomalous magnetic moment from lattice QCD", year = "2013", eprint = "1301.2607", archivePrefix = "arXiv", primaryClass = "hep-lat", SLACcitation $=$ "

[21] J. Prades, E. de Rafael, and A. Vainshtein arXiv:0901.0306 [hep-ph].

[22] Muon G-2 Collaboration, G. Bennett et al. Phys.Rev. D73 (206) 072003, arXiv:hep-ex/0602035 [hep-ex].

[23] B. Roberts Chin.Phys. C34 (2010) 741-744, arXiv:1001.2898 [hep-ex].

[24] M. Benayoun, P. David, L. DelBuono and F. Jegerlehner, arXiv:1210.7184 [hep-ph].

[25] J. Bijnens, E. Pallante, and J. Prades Nucl.Phys. B626 (2002) 410-411, arXiv: hep-ph/ 0112255 $[$ hep-ph]. 
[26] M. Hayakawa, T. Kinoshita, and A. Sanda Phys.Rev. D54 (1996) 3137-3153, arXiv:hep-ph/9601310 [hep-ph].

[27] M. Hayakawa and T. Kinoshita Phys.Rev. D57 (1998) 465-477, arXiv: hep-ph/ 9708227 [hep-ph].

[28] M. Hayakawa and T. Kinoshita arXiv:hep-ph/0112102 [hep-ph].

[29] A. Nyffeler PoS CD09 (2009) 080, arXiv:0912.1441 [hep-ph].

[30] A. Dorokhov, A. Radzhabov, and A. Zhevlakov Eur.Phys.J. C71 (2011) 1702, arXiv:1103. 2042 [hep-ph].

[31] K. T. Engel, H. H. Patel and M. J. Ramsey-Musolf, Phys.Rev. D86 (2012) 037502, arXiv:1201.0809 [hep-ph]

[32] P. Maris and C. D. Roberts Phys.Rev. C56 (1997) 3369-3383, arXiv: nucl-th/ 9708029 [nucl-th].

[33] P. Maris and P. C. Tandy Phys.Rev. C60 (1999) 055214, arXiv:nucl-th/9905056 [nucl-th].

[34] P. Maris and P. C. Tandy Phys.Rev. C61 (2000) 045202, arXiv:nucl-th/9910033 [nucl-th].

[35] D. Jarecke, P. Maris, and P. C. Tandy Phys.Rev. C67 (2003) 035202, arXiv:nucl-th/0208019 [nucl-th].

[36] P. Maris and P. C. Tandy Phys.Rev. C65 (2002) 045211, arXiv: nucl-th/ 0201017 [nucl-th].

[37] G. Eichmann, R. Alkofer, A. Krassnigg, and D. Nicmorus Phys.Rev.Lett. 104 (2010) 201601, arXiv:0912.2246 [hep-ph].

[38] G. Eichmann Phys.Rev. D84 (2011) 014014, arXiv:1104.4505 [hep-ph].

[39] G. Eichmann and C. S. Fischer, Eur. Phys. J. A 48 (2012) 9, arXiv:1111.2614 [hep-ph].

[40] H. Sanchis-Alepuz, G. Eichmann, S. Villalba-Chavez, and R. Alkofer Phys.Rev. D84 (2011) 096003, arXiv:1109.0199 [hep-ph]; H. Sanchis-Alepuz, R. Alkofer, G. Eichmann, and R. Williams PoS QCD-TNT-II (2011) 041, arXiv: 1112.3214 [hep-ph] ; H. Sanchis-Alepuz, R. Alkofer, and R. Williams PoS QNP2012 (2012) 112, arXiv:1206.6599 [hep-ph] .

[41] J. S. Bell, E. de Rafael, Nucl. Phys. B11 (1969) 611-620.

[42] R. Boughezal and K. Melnikov Phys.Lett. B704 (2011) 193-196, arXiv:1104.4510 [hep-ph].

[43] M. Della Morte, B. Jager, A. Juttner et al., [arXiv:1011.5793 [hep-lat]].

[44] J. Bijnens and J. Prades Z.Phys. C64 (1994) 475-494, arXiv: hep-ph/9403233 [hep-ph].

[45] J. S. Ball and T.-W. Chiu Phys.Rev. D22 (1980) 2542.

[46] C. S. Fischer, J. Phys. G 32 (2006) R253 [hep-ph/0605173].

[47] C. S. Fischer, D. Nickel, and J. Wambach Phys.Rev. D76 (2007) 094009, arXiv: 0705.4407 [hep-ph].

[48] T. Hahn Comput.Phys.Commun. 168 (2005) 78-95, arXiv:hep-ph/0404043 [hep-ph].

[49] P. Masjuan and M. Vanderhaeghen, arXiv:1212.0357 [hep-ph].

[50] A. A. Pivovarov, Phys. Atom. Nucl. 66, 902 (2003) [Yad. Fiz. 66, 934 (2003)] [hep-ph/0110248].

[51] M. J. Ramsey-Musolf, these proceedings. 